\title{
RESPUESTA A COMANDUCCI
}

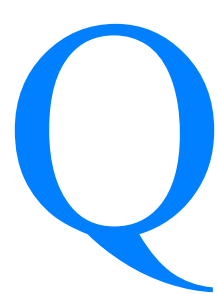

ue mi trabajo haya merecido la atención de un crítico a la vez tan generoso e incisivo como Paolo Comanducci es para mí un motivo de sincera satisfacción. Comanducci plantea con agudeza sus reservas hacia varios de los puntos fundamentales de mi argumentación; y esboza además una propuesta de «uso alternativo de la intuición de Raz» que, a su juicio, resulta más prometedora que el camino que yo he tomado. Eso es tanto como decir que con su estimulante y lúcido comentario replantea desde la raíz un debate que naturalmente debe proseguir y en el que mi aportación, como cualquiera habrá advertido con facilidad, tenía un carácter meramente exploratorio y tentativo. En esta nota, sin embargo, no pretendo desarrollar mis argumentos más allá de donde quedaron, con todas sus carencias, en el trabajo criticado por Comanducci: intentaré tan sólo contestar a sus objeciones estrictamente en los términos en que me las ha formulado.

En particular, no voy a adentrarme en la consideración de su propuesta alternativa, que en su comentario -tal y como yo lo entiendo- queda más apuntada o sugerida que verdaderamente desarrollada ${ }^{1}$. Me limitaré a hacer dos observaciones al respecto. La primera, que si bien Comanducci postula «una concepción menos cerrada de una regla como una razón excluyente» y nos sugiere «debilitar la conexión entre las reglas como razones excluyentes y el modelo basado en reglas» -sin que, dicho sea de paso, me quede del todo claro qué quiere decir «menos cerrada»y «debilitar»-, lo cierto es que, finalmente, el resto de sus observaciones críticas parecen ir dirigidas más bien a rehabilitar el concepto de razón excluyente en su original

1 Tampoco entraré a discutir su interpretación de Raz, que en los últimos tiempos empieza ya a ser objeto de una exégesis un tanto sofocante. Pero, en cualquier caso, no me parece que sea cierto que Raz no distinga entre el sentido explicativo y el sentido justificatorio de la expresión «razones para la acción»: vd. Raz, Practical Reason and Norms, Londres, Hutchinson, 1975. pp. 16 ss. 
y ya clásica acepción raziana, y no en algún nuevo sentido «menos cerrado» o «debilitado».

La segunda observación tiene que ver con su definición estipulativa de «regla»: «un enunciado-tipo normativo, emitido por el legislador y aún sin interpretar». Hay, desde luego, varios sentidos diferentes en los que hablamos de «reglas». Pero el que Comanducci ha elegido me parece que no es el relevante en este contexto, en el que se trata de discutir el papel específico de las reglas en los razonamientos prácticos. Yo diría que una «regla», en el sentido estipulado por Comanducci, no es sino una formulación normativa de la que se puede entender, dependiendo de las prácticas interpretativas que se desarrollen acerca de ella ${ }^{2}$, que expresa o bien un «principio» o bien una «regla» en algún otro sentido del término que queda pendiente de aclaración, que parece que es el relevante a la hora de distinguir entre diferentes tipos de estructura de un razonamiento práctico y que no puede ser, creo, el que corresponde a su estipulación.

En lo que sigue, por tanto, me centraré exclusivamente en las críticas que me formula Comanducci. Todas giran en torno a mi afirmación de que «un agente racional no puede en ningún caso aceptar reglas como razones excluyentes», lo que, según Comanducci, habría de crearme problemas en tres terrenos: I) «la explicación del razonamiento jurídico»; II) «la justificación de una conclusión en un razonamiento jurídico»; y III) «la teoría de la decisión racional en el ámbito jurídico». En los tres apartados siguientes intentaré responder a sus objeciones por ese mismo orden.

\section{I}

Comanducci llama la atención, en primer lugar, acerca del papel que de hecho desempeñarían las razones excluyentes en la explicación del razonamiento efectivo «de los jueces, abogados y ciudadanos». Su objeción consiste en que «generalmente las personas toman decisiones, en el campo jurídico, guiadas por reglas, y no por otros tipos de razones», lo que sería tanto como decir: a) «que no se guían por las mismas razones por las que generalmente lo hacen en campos que no pertenecen al dominio jurídico» (morales, prudenciales, etc.); y b) que «generalmente no ponderan, no balancean esas razones, si existe una regla jurídica y prescribe una conducta determinada».

Me parece oportuno formular dos observaciones preliminares antes de abordar la cuestión de fondo que encierra la objeción de Comanducci. En primer lugar, lo que sostiene Comanducci es una tesis de carácter empírico

${ }^{2}$ Sobre esta idea, en la que Schauer insiste particularmente -y a mi juicio con razón-, Vd. también, más recientemente, Cass R. Sunstein, «Problems with Rules», en California Law Review, 83 (1995) 953-1026, pp. $959-60$. 
acerca de los motivos -entendidos como estados mentales que son causas de acciones- que de hecho guiarían «generalmente» nuestras «decisiones en el campo jurídico». Como tal, resulta obvio que su afirmación -resulte o no correcta- no puede en modo alguno ser contradictoria con la que Comanducci llama mi tesis original: incluso si fuese verdad que «generalmente» aceptamos las reglas jurídicas como razones excluyentes, no veo en qué forma ello constituiría un argumento en contra de la afirmación de que un agente racional no podría hacer tal cosa (lo que, naturalmente, no es en absoluto una tesis empírica). Sería claramente posible dar la razón a Comanducci en cuanto a su pretensión descriptiva y seguir manteniendo a pesar de todo que, si eso es lo que ocurre en la práctica, nuestras prácticas son irracionales. Aunque decir esto me parece pertinente, no estoy sugiriendo, sin embargo, que en este punto hay entre nosotros un desacuerdo sólo aparente: hay un desacuerdo genuino, porque, como trataré de explicar en breve, discrepo además de la tesis descriptiva de Comanducci.

Antes de ello, una segunda observación que tiene ahora que ver con el alcance exacto de su tesis. Comanducci matiza sistemáticamente su pretensión con la cláusula «generalmente», porque, según admite, «algunas veces las reglas jurídicas no funcionan como razones excluyentes»; y esto sucedería «cuando las reglas jurídicas se apartan demasiado ${ }^{3}$ de otros tipos de razones para algunos agentes». El problema con esta matización, desde mi punto de vista, es que no queda suficientemente claro si la cláusula «generalmente» se refiere a los sujetos o a las ocasiones. Me da la impresión de que, tal y como la usa Comanducci, en realidad se refiere a ambas cosas. Y surge entonces una dificultad que acaso valga la pena comentar.

Lo que Comanducci afirma literalmente es que las reglas jurídicas no funcionan como razones excluyentes para un agente «cuando se apartan demasiado de otros tipos de razones» (o sea: que no funcionan para él como razones excluyentes en esas ocasiones; y se entendería entonces que sí en el resto). Lo que yo diría, en cambio, es que nunca funcionan para él como razones excluyentes si sólo está dispuesto a hacer lo que la regla prescribe a condición de que no «se aparte demasiado» del resultado del balance de razones, incluso si resulta que siempre (o casi siempre) cumple la regla porque nunca (o casi nunca) percibe ese apartamiento excesivo. Si la entiendo bien, la argumentación de Comanducci implica que sólo se puede decir que el agente «no decidió guiado por la regla» en casos en los que, finalmente, no hizo lo que la regla prescribía. Pero eso a mi juicio es una confusión: aquí lo relevante no es qué hizo el agente, sino por qué lo hizo.

Por eso estimo que la tesis de Comanducci -que a mí me parece ambigua- debe ser reinterpretada de uno de estos dos modos: o bien considera socialmente típica la actitud de quienes aceptan las reglas jurídicas como razones excluyentes y minoritaria o excepcional aquella otra -sustancialmente

${ }^{3}$ La cursiva es mía. 
diferente- de quienes «hacen lo que la regla prescribe mientras no se aparte demasiado de lo que exijan las razones aplicables al caso»; o bien considera socialmente típica esta última actitud, entendiendo que ello equivale a decir que sólo «generalmente»-y no «siempre», porque algunas veces sí que se produce ese apartamiento excesivo- las reglas jurídicas «funcionan como razones excluyentes» para quienes mantienen dicha actitud. En este segundo caso la cláusula «generalmente» se referiría a las ocasiones (y quizá también -pero esto no es ahora relevantea los sujetos); y la tesis resultante es a mi juicio rechazable por incurrir en la confusión anteriormente apuntada. En el primero, en cambio, se referiría sólo a los sujetos; y la tesis resultante sí me parece entonces -pero sólo entonces- perfectamente coherente.

Como es obvio, que sea coherente no implica que sea aceptable como tesis empírica. Ello nos lleva por fin a la cuestión de fondo: la plausibilidad de su pretensión descriptiva, interpretada del modo que ha quedado apuntado. Y creo que hay que empezar por aclarar qué es exactamente lo que se está pretendiendo describir.

Comanducci habla, en términos que se me antojan demasiado generales, de cómo «las personas toman decisiones en el campo jurídico», de cómo ha de explicarse el «razonamiento jurídico de los jueces, abogados y ciudadanos». Pero cuando hablamos de explicar un «razonamiento jurídico»o de cómo se toman «decisiones en el campo jurídico» podemos estar refiriéndonos -al menos- a dos cosas que convendría diferenciar ${ }^{4}$ : en primer lugar, al razonamiento práctico -como estricta actividad mental- que desarrolla un individuo cualquiera (operador jurídico o ciudadano privado) para decidir si hace o no lo que el derecho prescribe (incluso si, siendo un operador jurídico, lo que el derecho le ordena es dictar, motivándola, una determinada decisión); en segundo lugar, al razonamiento práctico -exteriorizado o verbalizadoque ofrece como motivación de sus decisiones el llamado por el derecho a adoptarlas (o en el caso del abogado: el que, con fines persuasivos, se presenta ante el decisor jurídico con la pretensión -sincera o no de que son ésas las consideraciones que deberían guiar su decisión). Huelga decir que los motivos reales de una decisión no tienen por que coincidir con los consignados en su motivación formal y expresa.

Me parece, por todo ello, que el problema de la «explicación del razonamiento jurídico» -o de «cómo se toman las decisiones en el campo jurídico»- debe desdoblarse en estas dos preguntas diferenciadas: 1) ¿por qué motivos los individuos hacen o dejan de hacer lo que el derecho les exige?; y 2) ¿qué es lo que los decisores jurídicos ofrecen como motivación de sus decisiones? Lo que he venido llamando «pretensión descriptiva» de Comanducci no es sino una respuesta única a estas dos preguntas: «generalmente, las

${ }^{4}$ El propio Comanducci ha trazado con suma claridad esta distinción en otro lugar: vid. Comanducci, Assaggi di metaetica, Torino, Giappichelli, 1992, pp. 199 ss. 
personas toman decisiones, en el campo jurídico, guiadas por reglas [como razones excluyentes], y no por otros tipos de razones». Veamos ahora, por separado, si es o no una respuesta adecuada para cada una de esas preguntas.

En cuanto a la primera, a mí me parece claro que diferentes personas, en diferentes situaciones y en relación con diferentes reglas jurídicas actúan de hecho por los motivos más variados: unas veces (como probablemente sucede con las normas de aparcamiento) nuestras decisiones pueden estar guiadas por meras consideraciones prudenciales; otras, por consideraciones morales en favor o en contra de lo que la regla exige, con absoluta independencia de que lo exija (la regla que tipifica y sanciona el homicidio ciertamente no es mi motivo para no matar; ni es el art. 110 del Código Civil mi motivo para «velar por mis hijos» $\mathrm{y}$ «prestarles alimentos»); y no se puede descartar que algunos individuos, en algunas situaciones y en relación con algunas reglas (y guiados probablemente por una concepción de la autoridad que a mí me parece irracional) actúen del modo en que lo hacen porque las aceptan como razones excluyentes. Pero la pretensión de que esto último es lo que sucede «generalmente» se me antoja gratuita y poco realista.

La tesis de Comanducci podría parecer entonces más acertada como respuesta a la segunda de las preguntas anteriormente diferenciadas. Pero tampoco en este caso me parece convincente. En definitiva lo que sostiene, traducido a la terminología de Schauer, es que («generalmente») los razonamientos jurídicos que desarrollan los órganos jurisdiccionales cuando motivan sus decisiones siguen de hecho la pauta del «modelo basado en reglas». Y yo sigo entendiendo que eso no es cierto.

Acepto desde luego que los jueces no motivan sus decisiones apelando a razones que ellos mismos reconozcan que son extrajurídicas: siempre tratan de sostener que sus pautas de decisión, aun cuando se trate de normas implícitas, tienen alguna clase de conexión o sustento en el derecho explícito que permite considerarlas dentro, y no fuera, de la esfera jurídica. Pero dejando al margen lo vaporosa que puede llegar a resultar esa «conexión» y lo indefinido que, en consecuencia, puede terminar siendo el borde de aquella esfera, dentro de ésta se entiende -me parece que de forma indisputada- que conviven habitantes de naturaleza diversa: no sólo reglas strictu sensu, sino también principios, esto es, razones -jurídicas- «de primer orden» que han de ser ponderadas o sopesadas para ser aplicadas y que se admite que pueden justificar contracciones o expansiones de la clase de casos prima facie gobernados por una regla (tal y como resultaría de la lectura literal de una disposición a la luz de las convenciones semánticas vigentes).

Me parece que el propio Comanducci está admitiendo todo esto cuando traza un paralelismo entre los modelos de Schauer y las distintas técnicas usuales de interpretación jurídica: el hecho de que todas ellas sean de utilización habitual basta a mi juicio para concluir -a pesar de la discrepancia de Comanducci- que el «modelo basado en reglas» no es una representación 
fiel del modo en que razonan de hecho los órganos jurisdiccionales cuando motivan sus decisiones.

\section{II}

En segundo lugar, Comanducci manifiesta ciertas reservas en lo tocante al uso del concepto de razón excluyente en la justificación de una conclusión en el razonamiento jurídico. Mi afirmación de que «un agente racional no puede en ningún caso aceptar reglas como razones excluyentes» le suscita tres dudas principales (que resumo alterando el orden en que él las expone): a) no queda del todo claro qué entiendo ahí por «racional»; b) en cualquier caso -y no sé hasta qué punto esta objeción es plenamente compatible con la anterior-, se trataría de una tesis puramente circular, una tautología que depende de mi propia estipulación acerca de lo que significa ser un agente racional; y c) esa estipulación constituiría una «elección moral última» sin fundamentación ulterior posible, tan última y tan imposible de fundamentar como otras elecciones morales alternativas (tales como el «legalismo» o el «deontologismo» morales): y no se ve, por consiguiente, por qué habría que aceptar que mi propia elección última es «mejor»o «más correcta» que cualquier otra de las opciones posibles.

Responder adecuadamente a cada una de estas objeciones requeriría mucho más espacio del que aquí puedo emplear (y me temo que también una claridad de ideas superior a la mía). Pero, al menos tentativamente, mis respuestas serían las siguientes.

a) Es indudable que el predicado «racional» se usa en muchos sentidos diferentes. Esto no es nada sorprendente, puesto que, en distintos contextos, se aplica a creencias (o a conjuntos de ellas), a preferencias de distintos tipos (o a conjuntos de ellas), a procedimientos de toma de decisiones (individuales o colectivos), a agentes, a acciones, etc. Esa aplicación, en ocasiones, puede violentar un tanto el uso ordinario del lenguaje. No es muy usual, p. ej., decir de una acción que es «racional» cuando se tiene en mente un sentido sustantivo de racionalidad, esto es, cuando lo que queremos decir es que hay razones -«todas las cosas consideradas»; o «no derrotadas»- para realizarla: en ese caso solemos decir, más bien, que la acción es «correcta» o que «está justificada». Pero cuando yo hablaba de un agente «racional» -o de uno que «actúa racionalmente»- no me refería a uno que realiza «acciones racionales» en ese sentido sustantivo que, en castellano ordinario, resulta decididamente extravagante: me refería a un agente que decide qué hacer de un modo racional. Que estas dos cosas no son idénticas, creo que resulta patente $^{5}$. Qué sentido le doy a la segunda es lo que quizá requiera alguna aclaración.

\footnotetext{
${ }^{5}$ Como ilustración de la diferencia entre estos dos sentidos, véase lo que escribió en su diario J.S. Mill (anotación correspondiente al 26 de febrero de 1854): "Carlyle dice de los ingleses que éstos actúan más racionalmente que la mayoría de los pueblos, pero que son más estúpidos que casi ningún otro pueblo en lo que se refiere a dar sus razones para ello»; y Mill apostilla de inmediato: «La segunda de estas proposiciones pone severos límites a la primera. Actuar bien sin saber decir por qué actúa uno así, es actuar bien sólo por accidente» (J.S. Mill, Diario, ed. de Carlos Mellizo, Madrid, Alianza, 1996, p. 38). Para Carlyle, los ingleses «actúan racionalmente» porque hacen lo que hay razones para hacer (i.e., la acción correcta o justificada); para Mill, en cambio, «no actúan racionalmente» porque no deciden racionalmente qué hacer (incluso si, «por accidente», terminan haciendo lo que es correcto o está justificado).
} 
Aunque a lo largo de la obra de Raz hay ciertas oscilaciones -e incluso pienso que incoherencias- a propósito de este asunto, he sostenido en otro $\operatorname{lugar}^{6}$ que el sentido en principio más aprovechable de la noción de «razón excluyente» tiene que ver con la idea de una estrategia indirecta para maximizar la probabilidad de actuar de conformidad con las razones aplicables al caso (y «excluidas» por la «razón excluyente» en cuestión). Decir entonces que un agente decide de modo racional -o, más brevemente, calificarle como «racional»- sería tanto como decir, en el sentido que aquí es relevante, que, dado un cierto conjunto de razones para actuar que él acepta - y que definen desde su punto de vista qué es lo correcto-, decide qué hacer siguiendo un procedimiento que verdaderamente maximiza la probabilidad de actuar de conformidad con dichas razones. Y decir, como sostuve, que «un agente racional no puede en ningún caso aceptar reglas como razones excluyentes», es decir que la aceptación de reglas como razones excluyentes no es ese procedimiento. Esto puede ser o no acertado, pero espero al menos que despeje las dudas en cuanto a qué entendía yo por «racional» en el contexto aludido.

b) Lo que se acaba de decir es relevante para responder a la segunda objeción (a saber: que incurro en petición de principio). Según Comanducci, la argumentación que ofrezco en apoyo de mi «tesis principal» tendría la siguiente estructura (que reconstruyo a partir de su texto con cierta libertad, pero espero que con fidelidad):

1) «un agente racional es aquel que actúa en concordancia con lo que debe hacer, y él debe hacer lo que, considerando todas las cosas, tiene que hacer»;

como «hacer o tomar una decisión considerando todas las cosas significa actuar sobre la base del balance de todas las razones relevantes», la premisa 1) equivaldría a esta otra:

1') un agente racional es aquel -y sólo aquel- que actúa sobre la base del balance de todas las razones relevantes,

y puesto que todos aceptamos que

2) «actuar sobre la base de una razón excluyente no es actuar considerando todas las cosas» (i.e., no es actuar sobre la base del balance de todas las razones relevantes),

entonces de 1) y 2) se seguiría mi «tesis principal»:

${ }^{6}$ Vid. J.C. Bayón, «Razones y reglas: Sobre el concepto de razón excluyente en J. Raz», en Doxa, 10 (1991) 25-66, págs. 43-49. 
3) no es un agente racional quien actúa sobre la base de una razón excluyente.

Lo que Comanducci me reprocha -como sucede siempre que se impute a alguien haber incurrido en petición de principio- no es que la inferencia sea inválida, sino que no aporta ningún argumento digno de consideración en favor de su conclusión, cuya verdad estaría simplemente presupuesta en las premisas 1) y su equivalente 1'). Esto es, que dadas 1) y 1') -junto con la no discutida 2)- ciertamente 3) es verdadera; pero verdadera sólo por definición, por una mera estipulación mía acerca de lo que significa ser un agente racional.

Si mi argumentación tuviera la estructura que ve en ella Comanducci, su circularidad sería indiscutible. Pero, por fortuna para mí, creo que no la tiene. Lo primero que me gustaría aclarar es si 1) y 1') son o no realmente mis premisas y si son o no realmente equivalentes. Para empezar por esto último, creo que 1) y 1') no hablan de lo mismo. La premisa 1) define al «agente racional» en el sentido de aquel que realiza «acciones racionales», entendiendo por tales -y aquí se trata, a mi modo de ver, de ese uso extravagante o no habitual de «racional» al que me referí antes- acciones correctas o justificadas. En cambio, la premisa 1') -que he de reconocer que Comanducci no formula expresamente, pero que creo que, implícitamente, me está atribuyendo-, define al agente racional en el sentido de aquel que decide qué hacer de un modo racional $^{7}$. La premisa 1) se refiere a actuar de conformidad con unas u otras razones y habla en realidad de las acciones del agente -cualesquiera que sean sus motivos- $\mathrm{y}$ de cuándo se las puede calificar como correctas; la 1'), a actuar por unas u otras razones, es decir, -nos habla de los motivos del agente y de cuándo se puede decir que fueron los correctos (incluso si el guiarse por ellos le lleva a realizar una acción que no es la correcta, porque un procedimiento de toma de decisiones puede ser el mejor en unas circunstancias dadas sin ser por ello infalible). Por eso, la conversión de 1) en 1') es engañosa: hacer lo que considerando todas las cosas se ha de hacer no es lo mismo que tomar una decisión considerando todas las cosas.

De hecho, me parece que la referencia en 1) al «agente racional» produce confusión. Lo que contiene en realidad 1), aunque expresado en forma un tanto oblicua, es una definición de la acción correcta o justificada. Y, estrictamente como tal, me parece bastante inofensiva y difícil de discutir. A menos, claro está, que uno entienda que las razones excluyentes tienen que ver con la determinación de qué acción es correcta y no, como pienso yo, con la de cuáles han de ser las consideraciones por las que el agente debe dejarse guiar -en ciertas circunstancias- a la hora de decidir qué hacer.

7 Por decirlo en los términos expuestos anteriormente -vid. nota 5-, la premisa 1) se refiere a «actuar racionalmente» en el sentido de Carlyle: la 1'), a «actuar racionalmente» en el sentido de Mill. 
De esto, en cambio, es exactamente de lo que nos habla 1' $)^{8}$. Lo que ocurre es que yo sí creo haber aportado algunos argumentos en favor de su aceptación, sin limitarme -como dice Comanducci- a haber estipulado crudamente que eso, y no otra cosa, es lo que hay que entender por «agente racional». Quizá convenga comentar qué tipo de argumentos eran esos y por qué me parecería injusto pedir otros de naturaleza diversa.

Uno de los problemas más fascinantes y que generan mayor complejidad dentro de una teoría de las razones para actuar viene dado por la pretensión de que, en determinadas circunstancias, intentar determinar de forma directa qué se ha de hacer resulta auto-frustrante, lo que es tanto como decir que en conjunto un agente se aproxima más a lo que tiene razones para hacer si utiliza una estrategia de decisión indirecta ${ }^{9}$. Ese es, por descontado, un problema ampliamente discutido en el seno de la tradición utilitarista, pero que puede generalizarse sin dificultad fuera de dicho marco. Pues bien, lo que ahora interesa es esto: a mi modo de ver, la naturaleza de esta pretensión es determinante a la hora de atribuir la carga de la prueba. No sé cómo se puede probar que el seguimiento de una estrategia directa en la toma de decisiones no es auto-frustrante: lo que en todo caso habrá que argumentar es que en ciertas circunstancias sí lo es y por qué; y que ciertas estrategias indirectas son, en esas circunstancias, globalmente preferibles. Y para quien ponga esto último en tela de juicio, no concibo más estrategia argumental que la puramente defensiva de intentar desmontar los argumentos aportados por quienes postulan la racionalidad de aquellas guías indirectas de la acción.

Raz ha proporcionado algunos argumentos de esa clase y yo he intentado explicar por qué no me parecen convincentes. Creo que ésa es toda la defensa posible de 1'). Y por eso me parece que el cargo de circularidad es injusto. Que finalmente 3) sea o no correcta no dependerá sólo de una «mera definición»: dependerá de la calidad -o de la falta de ella- de los argumentos que opuse a la pretensión de Raz.

c) La última objeción que Comanducci me formula en este apartado viene precedida por una pregunta que me parece paradójica («i[p]or qué no deberíamos ser “irracionales"?») ${ }^{10}$ y otra que creo que acaba de contestarse («ipor qué deberíamos aceptar [mi] definición de “racionalidad"?»). Quizá no esté de más, sin embargo, una precisión adicional. Yo también pienso que «es imposible encontrar una fundamentación moral última» (aunque ésta es, por descontado, una cuestión enormemente compleja sobre la que siempre

\footnotetext{
${ }^{8}$ Una premisa, por cierto, que sólo considero aceptable si se entiende que se refiere a lo que denominé un balance de razones refinado o esclarecido.

9 Vid. D. Parfit, Reasons and Persons, Oxford, Clarendon Press, 1984, pp. 55 ss.; y Larry Alexander, «Pursuing the Good -Indirectly», en Ethics, 95 (1985) 315-332.

${ }^{10}$ Prescindiendo de la doble negación, lo que Comanducci pregunta es por qué debemos ser racionales. Pero en mi opinión pedir razones para ser racional implica pretender situarse a la vez dentro y fuera de los límites del discurso relativo a las razones para actuar.
} 
es arriesgado pronunciarse sin un buen número de matizaciones que aquí no caben), pero no creo haber postulado ninguna «elección moral última» de carácter sustantivo. Creo, por el contrario, que la que Comanducci llama mi tesis principal es de naturaleza estrictamente formal (esto es, aspira a ser válida cualquiera que sea el contenido de las elecciones morales últimas de un agente); y ello, repito, porque entiendo que las razones excluyentes no tienen que ver con la determinación de qué acción es correcta, sino con la de cuál es el modo óptimo, en determinadas circunstancias, de decidir qué hacer ${ }^{11}$.

Me parece sin embargo -y probablemente ése es el origen último de nuestras discrepancias- que Comanducci entiende de un modo diferente la idea de razón excluyente. Y creo que esto resulta patente en los dos contraejemplos que opone a mi tesis principal como otras tantas «elecciones morales últimas» alternativas. De hecho, tal como los formula, estos contraejemplos me producen algún desconcierto. El primero de ellos, el «legalismo moral», queda definido como aquella posición según la cual «las reglas jurídicas son razones (morales) excluyentes para la acción»; el segundo, el «deontologismo moral», como aquella que postula «un sistema de reglas o principios morales, jerárquicamente ordenados, que son todos razones excluyentes de diferente grado». En cualquiera de esos dos casos, en realidad, parece que Comanducci entiende por razón excluyente o bien una que prevalece sobre otra (y que, en ese sentido, «excluye» el actuar con arreglo a ésta), o bien, simplemente, una que realmente es (considerada como) una razón para actuar (y que «excluye», comprensiblemente, el actuar con arreglo a lo que no se considera como tal). Pero para expresar cualquiera de esas dos ideas es del todo innecesario apelar a la noción de «razón excluyente», que por lo tanto estaría siendo utilizada de un modo espurio.

Si se desea evitar esa conclusión, pruébese a dar una respuesta diferente a las preguntas de cuáles serían, en cada uno de esos casos, las razones excluidas por cada una de esas presuntas razones excluyentes y qué otra cosa podría querer decir que quedan excluidas. Podría decirse, por ejemplo, que obedecer el derecho es, en conjunto, una estrategia más segura para hacer lo que se debe hacer que intentar determinar esto último por uno mismo (aunque no es eso lo que suele entenderse por «legalismo moral»o «positivismo ideológico», para el que o bien no hay más razón moral que las normas jurídicas, o bien estas últimas, reputadas como razones morales, prevalecerían en caso de conflicto sobre cualquier otra de signo contrario); o podría decirse también que seguir ciertas reglas morales es, en conjunto, una estrategia más segura para la maximización de lo bueno que intentar hacerlo

${ }^{11}$ Por supuesto se puede decir -y usualmente se dice- que un agente «ha hecho lo correcto» simplemente para indicar que, en las circunstancias en las que se hallaba, decidió qué hacer de modo irreprochable (i.e., sin que proceda imputarle responsabilidad por lo hecho), incluso si lo que hizo no fue la «acción correcta» (i.e., la que el agente hubiese reconocido como tal si hubiera podido decidir sin las limitaciones o constricciones con las que hubo de hacerlo). 
mediante el cálculo caso por caso (aunque no es eso lo que suele entenderse por «deontologismo», para el que o bien carece de sentido hablar de la maximización de lo bueno, o bien, al menos, lo correcto no se define como una variable dependiente de lo bueno). Pero entonces -y dejando al margen la carga de probar por qué serían estrategias más seguras- en ninguno de esos dos casos estaríamos hablando de «elecciones morales últimas», que es como las califica Comanducci: porque «últimas» serían las metas a las que supuestamente se llega mejor por esas vías indirectas; y en ningún caso dichas vías, que es de lo que, en esta posible interpretación alternativa, nos estarían hablando los contraejemplos de Comanducci y que no son de ninguna manera objeto de elección última, sino que se eligen con un fundamento: que conducen -presuntamente- de modo óptimo a metas que sí serían objeto de elección última. En suma: o bien Comanducci ha propuesto dos genuinos ejemplos de «elecciones morales últimas» en los que, sin embargo, la referencia a razones excluyentes me parece fuera de lugar; o bien la referencia en ellos a la idea de razones excluyentes es apropiada (aunque está por ver que sea aceptable), pero entonces no son genuinos ejemplos de «elecciones morales últimas».

\section{III}

Finalmente, un apunte muy breve acerca de la teoría de la decisión racional en el campo jurídico. Lo que Comanducci me reprocha en último lugar es que la tesis central que yo he mantenido resulta en la práctica paralizante y, por tanto, difícilmente defendible como criterio de decisión racional. En sus propias palabras, que «no podemos decidir siempre sobre la base de todas las razones relevantes, simplemente porque no tenemos tiempo suficiente»; y de ahí se seguiría que, «después de todo, tenemos que actuar de acuerdo a la elección de reglas como razones excluyentes» ${ }^{12}$.

Creo, sin embargo, que entre esas dos afirmaciones hay un salto considerable. Estoy totalmente de acuerdo en que un agente verdaderamente racional debe tomar en serio sus deficiencias epistémicas y los costes de adquirir y procesar información, lo que seguramente equivale a reconocer que «no podemos decidir siempre sobre la base de todas las razones relevantes»

${ }^{12}$ Comanducci aduce también, en apoyo de esta conclusión, que la superioridad del utilitarismo de la regla sobre el utilitarismo del acto ha sido mostrada por algunos teóricos de la decisión racional. Quizá esté pensando en autores como Harsanyi, de cuya obra es un excelente conocedor: vid. P. Comanducci, Contrattualismo, utilitarismo, garanzie ( $2^{\mathrm{a}}$ ed.), Torino, Giappichelli, 1991. Pero ésta es sin duda una cuestión polémica y extraordinariamente compleja: y creo que Comanducci me concederá que, sin entrar a desarrollar los argumentos pertinentes -cosa que ni él ni yo podemos hacer en este momento-, resulta difícil convencer de la corrección de uno cualquiera de los puntos de vista posibles a quien no esté ya convencido de antemano. 
(es decir, sobre la base de lo que yo llamé un balance «tosco» o «no refinado»). Pero Comanducci ha dado por sentado que la única alternativa posible es entonces actuar de acuerdo a la elección de reglas como razones excluyentes. Y esto último es exactamente lo que yo discutía, proponiendo en su lugar como estrategia de decisión más racional el recurso a «reglas indicativas» en el sentido de Regan. Quizá la noción de regla indicativa esté mal concebida. Quizá el procedimiento de decisión basado en reglas indicativas, rectamente entendido, resulte en realidad extensionalmente equivalente al basado en el seguimiento de reglas como razones excluyentes. Pero Comanducci debería haber aportado algún argumento que sustentara estas -u otras- formas posibles de crítica. En su ausencia, sigo pensando que la tesis que sostuve no ha sido aún desacreditada. 